\title{
Infantile-Onset Saccade Initiation Delay in a Child with a Thin Intercollicular Commissure
}

\author{
Michael S. Salman, Kristin M. Ikeda, Jens Wrogemann
}

Can. J. Neurol. Sci. 2010; 37: 893-896

Infantile-onset saccade initiation delay (ISID), also known as congenital ocular motor apraxia, is an uncommon condition characterized by an inability to trigger horizontal volitional saccades. ${ }^{1-3}$ Vertical saccades and smooth ocular pursuit are usually preserved. ${ }^{1,3}$ Reflexive saccades may be impaired. ${ }^{1,4-6}$ Saccade initiation delay is a clinical finding and not a specific disease. ${ }^{7}$ The diagnosis is usually made after head control is achieved in infants between four to six months-of-age, when characteristic head thrusts appear. The infant initially makes use of head thrusts and later on uses eye blinks to rapidly shift the direction of gaze to overcome the difficulty in initiating saccades. ${ }^{8,9}$ Infantile-onset saccade initiation delay is often associated with developmental delay in the areas of fine motor, gross motor, speech, and language development. ${ }^{3,8,10-14}$

Neuroradiological findings in ISID are diverse and include various abnormalities involving hindbrain structures or agenesis of the corpus callosum. ${ }^{3,6,13-17}$ The etiology of ISID is unknown, but it has been suggested that the abnormality lies in the midbrain, ${ }^{3}$ or cerebellum. ${ }^{8,15}$ In this paper we present a child who has difficulties initiating horizontal saccades and describe his videotaped eye movements and unique brain MRI findings in detail. We then suggest a mechanism for the pathogenesis of saccade initiation delay.

\section{CASE Report}

A three-year-old boy was referred for an assessment of his abnormal head movements. He was noted to make horizontal head thrusts when looking at objects since the age of one year. Pregnancy and delivery at full term were normal. He sat at eight months and walked at 18 months, suggesting mild motor delay. His language development was also delayed. He spoke his first words at 18 months and made two-word sentences at 30 months. Past medical history was significant for iron-deficiency anemia at age two years, which was treated with iron. The family is Aboriginal Canadian from Northern Ontario. His mother is Ojibway-Cree and his father is Ojibway. No relevant family history was elicited.

On initial assessment, his general physical exam revealed no neurocutaneous stigmata or dysmorphic features. Visual acuity was normal bilaterally. Pupils were equal and reactive to light. His fundi were normal. He used intermittent horizontal head thrusts when asked to look at objects to the right or left of his visual fields. He was noted to make spontaneous small amplitude saccades intermittently in all directions. Horizontal smooth pursuit was slightly saccadic but within normal limits for age. Vertical saccades and smooth pursuit were normal. The oculocephalic maneuver (low frequency vestibulo-ocular reflex)

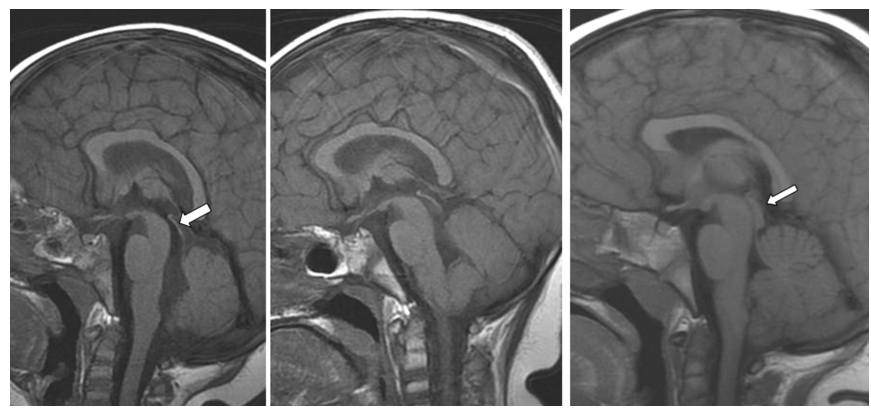

Figure 1: Midsagittal T1-weighted brain MRI of the patient at 3 yearsof-age (left), at 6 years-of-age (centre), and age-matched normal control at 6 years-of-age (right). There is severe thinning of the intercollicular commissure in the patient in comparison to the control (arrows).

showed full range of normal smooth eye movements both vertically and horizontally. The rest of the cranial nerve exam was normal. Tone, power, coordination, reflexes, and gait were also normal. His developmental assessment revealed mild delays in his speech and language, fine motor, and visual-motor domains.

Brain MRI showed an almost absent intercollicular commissure. There was only a thin band of tissue connecting the right and left superior and inferior colliculi. The superior and inferior colliculi were otherwise normal. There was no cleft. Other brain structures, including the cerebellum and corpus callosum, were normal (Figures 1-3).

At four and a half years-of-age, the patient continued to use head thrusts to initiate horizontal saccades. The rest of the examination remained unchanged. His speech and language development was delayed. On follow-up at five years and nine months-of-age, we undertook a more detailed examination of his

\footnotetext{
From the Section of Pediatric Neurology (MSS), Children's Hospital; Section of Pediatric Radiology (JW), Department of Radiology, Health Sciences Centre, University of Manitoba (MSS, JW), Winnipeg, Manitoba; Faculty of Health Sciences (KMI), Michael G. DeGroote School of Medicine, McMaster University, Hamilton, Ontario, Canada.

ReCeived OCtober 6, 2009. Final Revisions Submitted January 14, 2010. Correspondence to: Michael S. Salman, Section of Pediatric Neurology, Children's Hospital, AE 308, 820 Sherbrook Street, Winnipeg, Manitoba, R3A 1R9, Canada.
} 


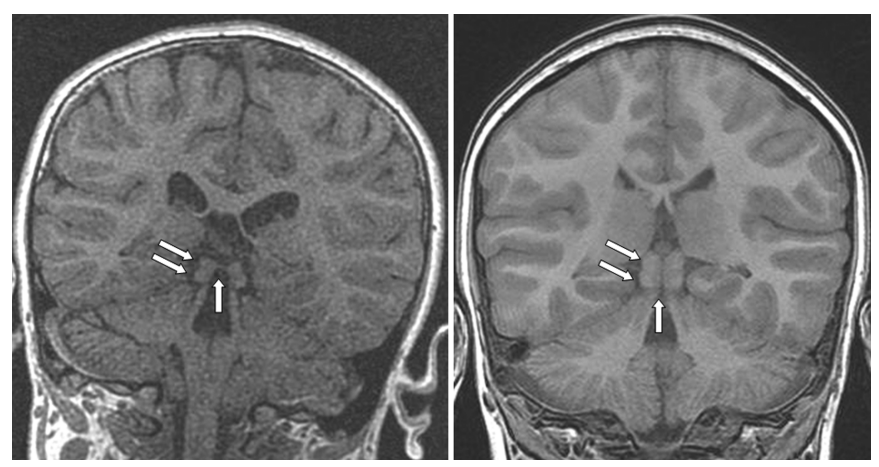

Figure 2: Coronal T1-weighted fast spolied gradient-echo (FSPGR) brain MRI of the patient through the tectum at age 6 years (left) and agematched normal control (right). There is a thin band of tissue connecting the right and the left superior colliculi in the patient in comparison to the control (vertical arrow). The superior (upper arrows) and inferior (lower arrows) colliculi appear normal otherwise.

eye movements and videotaped them (See video clip on-line). He was unable to initiate horizontal saccades to command, unpredictable visual stimuli, auditory stimuli, somatosensory stimuli (touch), or look alternately between two targets, i.e. internally generated saccades. In order to fixate on the target, he would thrust his head towards the target, blink, or do both. Head thrusts occurred less frequently in comparison with his initial assessment. When his head was fixed, he would blink to initiate horizontal saccades. The horizontal saccadic latency was prolonged. Horizontal smooth ocular pursuit was normal for age. His vertical eye movements were normal. The small field optokinetic response, using a rotating drum held $15 \mathrm{~cm}$ in front of his eyes, was absent in all directions. When we reviewed the videotape of his eye movements frame-by-frame, we found that in addition to utilizing the vestibulo-ocular reflex to drive his eyes contralaterally to the direction of the head thrust as described in many reports, $, 1,10,14,17$ he also moved his eyes rapidly in the same direction as the head thrusts. This was associated with eye blinks on several occasions. Developmental re-assessment showed that he had a variety of abilities that were slightly below his age level, in the 5-5.5 year range, including mild delays in fine and gross motor skills. Investigations including lipid profile, albumin, liver enzymes, $\alpha$-fetoprotein, and CBC were normal. Repeat brain MRI revealed no changes.

At age seven years, his head thrusts were reported to be occurring less frequently. His examination remained unchanged. The horizontal and vertical fast phases of the optokinetic response were still impaired. The fast phases were absent when he was spun horizontally in a rotating chair in either direction. His eyes locked up in extreme gaze contralateral to the direction of the spin. The horizontal vestibulo-ocular reflex cancellation test was normal. This was assessed by asking him to look at his right thumb nail while spinning him on a rotating chair. The eyes remained in the orbital mid position without nystagmus during the test.

To assess whether his impaired saccade initiation was caused by an inability to break visual fixation, he was seated opposite a featureless wall in a dim lit room with his head fixed. Visual fixation was eliminated further by placing a +20 lens in front of each eye or a 'one-way' lens over one eye while occluding the other eye. This one-way lens allows the examiner to see the eyes while the subject is unable to see through the lens. The patient was then asked to look to the right or left. He either blinked to make horizontal saccades or generated slow eye movements, demonstrating that his saccade initiation difficulties persisted in the absence of visual fixation.

When he was asked not to blink or to hold his eyelids open with his fingers to eliminate any blinks and then look to the right or left with his head fixed, he could only generate slow eye movements.

\section{Discussion}

We present a young child with a clinical presentation of ISID, characterized by an inability to initiate horizontal saccades on command or reflexively, normal horizontal smooth pursuit, and normal vertical eye movements. Our patient used horizontal head thrusts or blinks, and occasionally both head thrusts and blinks to initiate horizontal saccades. Infantile-onset saccade initiation delay persists with age but the predominant strategy used to initiate saccades changes from head thrusts to blinks. Our patient's inability to initiate horizontal saccades persisted during a task that eliminated visual fixation. This is consistent with findings from a report in which the difficulty in initiating saccades persisted in the dark in patients with ISID. ${ }^{5}$ Therefore, the inability to break visual fixation does not account for ISID.

When horizontal saccades in children with ISID are recorded, they demonstrate increased saccadic latency and hypometric saccades. Saccadic velocity is typically normal. ${ }^{5,18} \mathrm{We}$ agree that the term 'ocular motor apraxia' is inappropriate in patients who cannot generate volitional and reflexive saccades, ${ }^{2}$ because the term apraxia refers to a defect of volitional movements with preservation of reflexive movements.

The neuroradiological finding in our patient is unique. The most common radiological abnormality found in ISID is cerebellar hypoplasia, especially of the inferior vermis. . $^{3,4,6,8,13-15,17}$
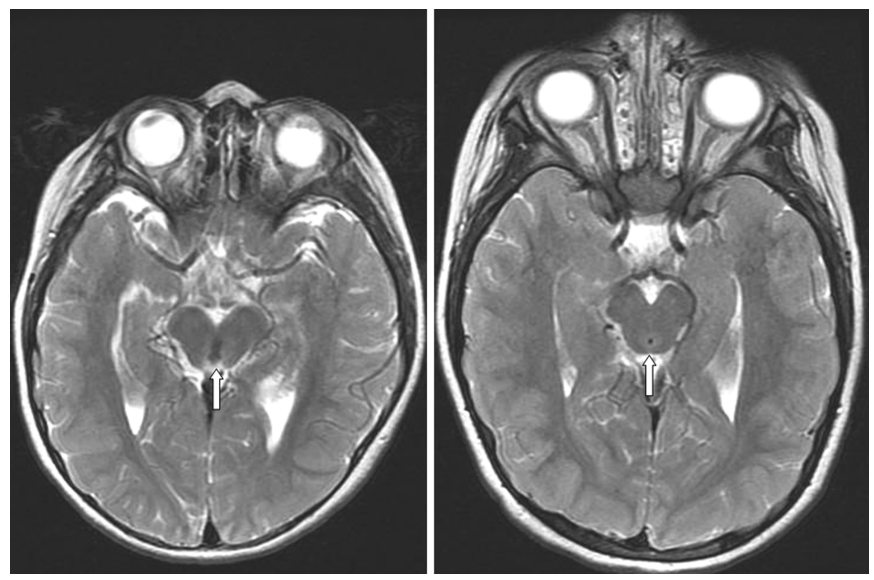

Figure 3: Axial T2-weighted brain MRI of the patient through tectum and cerebral aqueduct at age 6 years (left) and age-matched normal control (right). The intercollicular commissure is very thin (arrows). 
The axial and coronal MRI images in our case show that the superior and inferior colliculi are intact, but the intercollicular commissure that normally connects the right and left side, is abnormally thin. The origin of this abnormality is not likely to be the result of a vascular accident and more likely to be genetic, caused by a defective gene involved in axonal guidance and decussation (Dr. Harvey Sarnat, personal communication). Absence of the intercollicular sulcus, which separates the superior and inferior colliculi, has been reported in a child with ISID. ${ }^{17}$ It resulted in a fused appearance of the superior and inferior colliculi. No mention was made about a lack of connections between the superior colliculi in that report.

The intercollicular commissure connects roughly similar regions between the left and right colliculi i.e., fibers from one region of the superior colliculus have been found to project to the same area of the contralateral colliculus in humans. ${ }^{19}$ The function of the connections between colliculi remains poorly understood, but it has been suggested that these connections may be involved in more complex aspects of visual processing; for example, orientating gaze, attention, and transferring visual information. ${ }^{19}$

The superior colliculus exerts an important role in processing saccades.$^{20,21}$ Lesions in the superior colliculus cause hypometric saccades with increased saccadic latency in monkeys. ${ }^{20,22}$ However, lesions in this area do not result in permanent difficulties. ${ }^{22}$ The superior colliculi appeared normal in our patient. The almost complete absence of the intercollicular commissural fibers and the resultant impaired communication between analogous structures within the superior colliculi likely caused the difficulties in initiating horizontal saccades in our case. We suggest that a similar mechanism could also explain ISID in patients with cerebellar vermis hypoplasia and in agenesis or hypoplasia of the corpus callosum..$^{2,7,10,23}$ We propose that the fundamental problem underlying saccade initiation delay is a congenital or an acquired disruption or disconnection of axons linking analogous brain regions involved in processing saccades (See the accompanying article on page 779 for further details).

\section{Blinks and saccade initiation delay}

There is bilateral activation of the frontal eye field (FEF) on fMRI in conjunction with blinks, which occurs independently of saccades. ${ }^{24}$ Saccades are commonly accompanied by blinks. ${ }^{21}$ The use of blinks to facilitate saccades in children with ISID is well documented, especially as children get older, implying that it may be a learned response. ${ }^{6,10}$ Intentional blinking and saccades appear to activate slightly different areas within the FEF; however, the same areas of the FEF are activated during both saccades and blinks, and this may coordinate the two activities. ${ }^{25}$ We propose that bilateral activation of the FEF using blinks may facilitate the generation of volitional saccades in patients with ISID. The resultant signal can then be transferred to the brainstem to trigger saccades through direct neuronal connections between the FEF and the brainstem. ${ }^{21}$

Blinks may facilitate saccadic initiation through another mechanism. Pause cells of the paramedian pontine reticular formation stop firing during blinks. ${ }^{26}$ Cessation of pause cells' tonic discharge normally occurs during saccades, and the cooccurrence of blinking with saccades may further facilitate saccadic initiation in patients who are otherwise unable to initiate saccades. ${ }^{6}$ Blinks also reduce the latency of head movement. ${ }^{27}$ This may explain the strategy of combining blinks and head movements in our patient to perhaps make saccade initiation more efficient.

\section{Head thrusts and saccade initiation delay}

Rapid movement of the eyes in the same direction as the head thrust was noted in our patient. It is easy to miss this sign on examination, especially when blinks occur at the same time as the head thrusts. We only saw it when we reviewed the videotape of the eye movement examination frame-by-frame. This finding has been reported rarely in children, ${ }^{5}$ and in two adults with ocular motor apraxia. ${ }^{18}$ In both papers, the authors found that saccades and head thrusts occurred almost simultaneously. Head movement regions are located very close to the FEF. ${ }^{28}$ It has also been demonstrated that stimulation of the dorsomedial FEF in monkeys results in eye and head movements that shift gaze. ${ }^{29}$ Stimulation of the areas which induced head movements always resulted in some form of eye movement, either saccadic or a counter-rotation movement. ${ }^{28}$ In addition, an increased duration of FEF stimulation resulted in staircase movement gaze shifts with one large head movement. ${ }^{29}$ Head and eye movements are coordinated in the $\mathrm{FEF}^{29}$ We suggest that head thrusts in patients with ISID may facilitate the initiation of saccades through activating head movement neurons within the FEF. The vestibulo-ocular reflex, which usually drives the eyes contralateral to the direction of the head movement, is partially disengaged, ${ }^{21}$ or suppressed, ${ }^{18}$ when the head and eyes move in the same direction.

\section{Developmental delay and saccade initiation delay}

The developmental delay in our case is consistent with that previously reported in the literature,,$^{3,8,10-14}$ consisting of mild delay in gross motor, fine motor, visual motor, speech, and language domains. It may be related to the lack of the intercollicular commissure and the resultant impaired communication. This connection is likely required for the proper integration of somatosensory, visual, and auditory information. However, additional brain abnormalities, not apparent on his MRI, may also be responsible for his mild developmental delay.

\section{REFERENCES}

1. Cogan DG. A type of congenital ocular motor apraxia presenting jerky head movements. Trans Am Acad Ophthalmol Otolaryngol. 1952;56:853-62.

2. Harris CM, Shawkat F, Russell-Eggitt I, Wilson J, Taylor D. Intermittent horizontal saccade failure ('ocular motor apraxia') in children. Br J Ophthalmol. 1996;80:151-8.

3. Kondo A, Saito Y, Floricel F, Maegaki Y, Ohno K. Congenital ocular motor apraxia: clinical and neuroradiological findings, and long-term intellectual prognosis. Brain Dev. 2007;29:431-8.

4. Harris CM, Hodgkins PR, Kriss A, Chong WK, Thompson DA, Mezey LE, et al. Familial congenital saccade initiation failure and isolated cerebellar vermis hypoplasia. Dev Med Child Neurol. 1998;40:775-9.

5. Zee DS, Yee RD, Singer HS. Congenital ocular motor apraxia. Brain. 1977; 100:581-99.

6. Shawkat FS, Kingsley D, Kendall B, Russell-Eggitt I, Taylor DSI, Harris CM. Neuroradiological and eye movement correlates in children with intermittent saccade failure: "ocular motor apraxia". Neuropediatrics. 1995;26:298-305. 
7. PeBenito R, Cracco J. Congenital ocular motor apraxia: case reports and literature review. Clin Pediatr (Phila). 1988;27(1): 27-31.

8. Jan JE, Kearney S, Groenveld M, Sargent MA, Poskitt, KJ. Speech, cognition and imaging studies in congenital ocular motor apraxia. Dev Med Child Neurol. 1998;40:95-9.

9. Summers CG, MacDonald T, Wirtschafter JD. Ocular motor apraxia associated with intracranial lipoma. J Pediatr Ophthalmol Strabismus. 1987;24(5):267-9.

10. Fielder AR, Gresty MA, Dodd KL, Mellor DH, Levene MI. Congenital ocular motor apraxia. Trans Ophthalmol Soc UK. 1986;105:589-98.

11. Rappaport L, Urion D, Strand K, Fulton AB. Concurrence of congenital ocular motor apraxia and other motor problems: an expanded syndrome. Dev Med Child Neurol. 1987;29:85-90.

12. Anteby I, Lee B, Noetzel M, Tychsen L. Variants of congenital ocular motor apraxia: associations with hydrocephalus, pontocerebellar tumor, and a deficit of vertical saccades. JAAPOS. 1997;1:201-8.

13. Kim JS, Park SH, Lee KW. Spasmus nutans and congenital ocular motor apraxia with cerebellar vermian hypoplasia. Arch Neurol. 2003;60:1621-4.

14. Marr JE, Green SH, Willshaw HE. Neurodevelopmental implications of ocular motor apraxia. Dev Med Child Neurol. 2005;47:815-9.

15. Sargent MA, Poskitt KJ, Jan JE. Congenital ocular motor apraxia: imaging findings. Am J Neuroradiol. 1997;18:1915-22.

16. Borchert MS, Sadun AA, Sommers JD, Wright KW. Congenital ocular motor apraxia in twins. J Clin Neuroophthalmol. 1987;7(2):104-7.

17. Whitsel EA, Castillo M, D'Cruz D. Cerebellar vermis and midbrain dysgenesis in oculomotor apraxia: MR findings. Am J Neuroradiol. 1995;16:831-4.

18. Rambold H, Moser A, Zurowski B, Gbadamosi J, Kömpf D, Sprenger A, et al. Saccade initiation in ocular motor apraxia. J Neurol. 2006;253:950-2.
19. Tardif E, Clarke S. Commissural connections of human superior colliculus. Neurosci. 2002;111(2):363-72.

20. Schiller PH, True SD, Conway JL. Deficits in eye movements following frontal eye-field and superior colliculus ablations. J Neurophysiol. 1980;44:1175-89.

21. Leigh RJ, Zee, DS. The neurology of eye movements. 4th ed. New York: Oxford University Press; 2006.

22. Mohler CW, Wurtz RH. Role of striate cortex and superior colliculus in visual guidance of saccadic eye movements in monkeys. J Neurophysiol. 1977;40:74-94.

23. Orrison WW, Robertson WC. Congenital ocular motor apraxia: a possible disconnection syndrome. Arch Neurol. 1979;36:29-31.

24. Bodis-Wollner I, Bucher SF, Seelos KC. Cortical activation patterns during voluntary blinks and voluntary saccades. Neurology. 1999;53(8):1800-5.

25. Kato M, Miyauchi S. Human precentral cortical activation patterns during saccade tasks: an fMRI comparison with activation during intentional eyeblink tasks. Neuroimage. 2003;19: 1260-72.

26. Mays LE, Morrisse DW. Electrical stimulation of the pontine omnipause area inhibits eye blink. J Am Optom Assoc. 1995;66(7):419-22.

27. Evinger C, Manning KA, Pellegrini JJ, Basso MA, Powers AS, Sibony PA. Not looking while leaping: the linkage of blinking and saccadic gaze shifts. Exp Brain Res. 1994;100(2):337-44.

28. Gagnon D, O'Driscoll GA, Petrides M, Pike GB. The effect of spatial and temporal information on saccades and neural activity in oculomotor structures. Brain. 2002;125:123-39.

29. Knight TA, Fuchs AF. Contribution of the frontal eye field to gaze shifts in the head-unrestrained monkey: effects of microstimulation. J Neurophysiol. 2007;97:618-34. 